\title{
INFLUÊNCIA DO CLIMA NA INCIDÊNCIA DE INFECÇÃO RESPIRATÓRIA AGUDA EM CRIANÇAS NOS MUNICÍPIOS DE CAMPINA GRANDE E MONTEIRO, PARAÍBA, BRASIL
}

\author{
JULLIANNA VITORIO VIEIRA DE AZEVEDO ${ }^{1}$, CARLOS ANTONIO COSTA DOS SANTOS ${ }^{1}$, \\ TELMA LUCIA BEZERRA ALVES ${ }^{1}$, PEDRO VIEIRA DE AZEVEDO ${ }^{1}$, RICARDO ALVES DE OLINDA ${ }^{2}$ \\ ${ }^{1}$ Programa de Pós Graduação em Recursos Naturais (PPGRN), Universidade Federal de Campina Grande \\ (UFCG), Campina Grande, PB, Brasil \\ ${ }^{2}$ Universidade Estadual da Paraíba, Departamento de Estatística, Campina Grande, PB, Brasil
}

julliannavitorio@hotmail.com carlos.santos@ufcg.edu.br; telmalu@yahoo.com.br; azevedopedrovieira@ gmail.com, ricardo.estat@yahoo.com.br

Recebido Maio de 2014 - Aceito Fevereiro de 2015

\begin{abstract}
RESUMO
O presente estudo avaliou a influência das variáveis climáticas (precipitação pluvial, temperatura e umidade do ar) na incidência de infecções respiratórias agudas (IRA) em crianças menores de dois anos nos municípios de Monteiro e Campina Grande, na Paraíba, no período de 1998 a 2012. A correlação linear múltipla foi utilizada na avaliação do grau de associação entre as variáveis climáticas e o número de casos de IRA. Os resultados mostram que, para Campina Grande, evidenciou-se comportamento quadrático da variável IRA em crianças menores de dois anos, tendo ocorrido o máximo desses casos entre os anos de 2003 e 2005. No entanto, para Monteiro, o número anual de ocorrência de casos de IRA é bastante variável e irregular. Em Campina Grande, apenas a temperatura e a umidade relativa do ar foram significativas para representar a incidência de IRA em crianças menores de 2 anos, com $99 \%$ da variabilidade explicada pelo modelo. Já para Monteiro, a análise mostra que as três variáveis climáticas foram significativas para representar a incidência dessas infecções, com $90 \%$ da variabilidade explicada pelo modelo.
\end{abstract}

Palavras-chave: doenças respiratórias, precipitação pluvial, temperatura e umidade relativa do ar.

\begin{abstract}
INFLUENCE OF CLIMATE ON THE INCIDENCE OF ACUTE RESPIRATORY INFECTIONS IN CHILDREN AT THE DISTRICTS OF CAMPINA GRANDE AND MONTEIRO, PARAÍBA, BRAZIL

The present study evaluated the influence of climatic variables (rainfall, air temperature and humidity) on the incidence of Acute Respiratory Infections (ARI) in children under two years old in the counties of Monteiro and Campina Grande, Paraíba, during the period from 1998 to 2012. A multiple linear correlation model was used to assess relationship between climate variables and the number of cases of ARI. The results for Campina Grande, revealed a quadratic behavior of the IRA variable in children under two years old and the maximum occurrence of these cases between the years 2003 and 2005. However, at Monteiro, the annual number of occurrence of ARI cases is highly variable and irregular. In Campina Grande, only temperature and relative humidity were significant to represent the incidence of ARI in children younger than 2 years, with $99 \%$ of the variability explained by the model. However, at Monteiro, the analysis shows that the three climate variables were significant for representing the incidence of these infections with $90 \%$ of the variability explained by the model.

Keywords: respiratory diseases, rainfall, air temperature and relative humidity.
\end{abstract}




\section{INTRODUÇÃO}

$\mathrm{O}$ vírus sincicial respiratório (VSR) é o agente viral mais importante causando doença respiratória grave em lactentes e crianças em todo o mundo (Meerhoff et al., 2009). A maioria das crianças são infectadas pelo menos uma vez antes de completar 2 anos e reinfectadas ao longo da vida. Os sintomas de uma infecção por VSR variam de uma doença muito leve a infecções respiratórias graves do trato respiratório, incluindo bronquiolite e pneumonia. Embora raramente reconhecido, o VSR é comum em adultos e pode ser a causa de uma doença grave, especialmente nos idosos. A taxa média anual de hospitalização associada à VSR tem sido relatada de 3 (intervalo: 2-6) por 1000 crianças e de 17 (intervalo: 12-34) por 1000 crianças com idade inferior a 6 meses. Entretanto, a mortalidade em crianças é baixa (8,4 por 100.000 habitantes). Mais da metade das mortes atribuídas à crianças ocorre em bebês com idade entre 1 a 12 meses.

Em 2002, as infecções respiratórias agudas (IRA) foram responsáveis por 3,96 milhões de mortes de crianças, sendo que até $95 \%$ se devem à doença do trato respiratório inferior (DTRI). Dois terços dessas mortes ocorrem em menores de 1 ano de idade, particularmente $(90 \%)$ em países em desenvolvimento (OMS, 2002). Atualmente, as infecções respiratórias agudas (IRA) continuam a ser a principal causa de morbidade e mortalidade em crianças menores de cinco anos em todo o mundo, respondendo por cerca de dois milhões de mortes por ano. As populações com maiores riscos de desenvolver uma doença respiratória fatal são os jovens, os idosos e os imunodeprimidos. Embora as infecções das vias respiratórias superiores (IVRS) sejam muito frequentes, mas raramente com risco de vida, as infecções das vias respiratórias inferiores (IVRI) são responsáveis por doenças mais graves, tais como: gripe, pneumonia, tuberculose e bronquiolite, que são os principais contribuintes para a mortalidade por IRA. A incidência de IRA nas crianças com menos de 5 anos é estimada em 0,29 e 0,05 episódios por criança/ano nos países em desenvolvimento e industrializados, que traduz-se em 151 milhões e 5 milhões de novos episódios a cada ano, respectivamente (Rudan et al., 2008). A maior incidência ocorre nos países em desenvolvimento e subdesenvolvidos: Índia (43 milhões), China (21 milhões), Paquistão (10 milhões), Bangladesh, Indonésia e Nigéria (56 milhões cada). A pneumonia é responsável por $21 \%$ de todas as mortes em crianças menores de 5 anos, levando-se a estimar que de cada mil crianças nascidas vivas, 12 a 20 morrem por pneumonia antes do seu quinto aniversário. Os principais agentes etiológicos responsáveis por IRA em crianças incluem: Streptococcus pneumoniae, Haemophilus influenzae tipo b (Hib), Staphylococcus aureus e outras espécies bacterianas, VSR, vírus do sarampo, vírus da parainfluenza humana de tipo
1, 2 e 3 (PIV - 1, PIV - 2 e PIV - 3), vírus influenza e vírus da varicela. Estima-se que $90 \%$ das IRAs são relacionadas com agentes virais (OMS, 2002; Benguigui, 2002; OMS, 2004).

Convém ressaltar que o organismo infantil possui algumas particularidades que devem ser consideradas. A relação entre superfície corporal e peso é 2,5 vezes maior nos neonatos do que nos adultos, o que leva a uma maior área de perda de calor por unidade de peso. Tal fato, associado a uma maior velocidade de crescimento, gera taxas de metabolismo em repouso e de consumos de oxigênio por quilo de peso mais elevadas nas crianças do que nos adultos. Em condições de repouso e equilíbrio térmico, um lactente consome $7 \mathrm{ml} \cdot \mathrm{kg}^{-1}$. $\min ^{-1}$ de oxigênio, contra $3,5 \mathrm{ml} \cdot \mathrm{kg}^{-1} \cdot \mathrm{min}^{-1}$ nos adultos, nas mesmas condições. $\mathrm{O}$ volume de ar que passa pelos pulmões de um lactente é, assim, duas vezes maior que o de um adulto em repouso, por unidade de peso corporal. Isso faz com que qualquer agente químico ou variação nas condições atmosféricas atinja duas vezes mais as vias respiratórias de uma criança entre uma semana e doze meses de idade, se comparadas as de um adulto no mesmo período de tempo.

Quando a temperatura ambiente cai abaixo do nível do equilíbrio térmico, aumentam a velocidade do metabolismo e as necessidades de oxigênio. Como a temperatura basal dos lactentes é superior a dos adultos, uma queda igual de temperatura provoca maior consumo de oxigênio nas crianças, que também necessitam de mais oxigênio devido ao choro (Andreoli et al., 1993; Cardoso, 2007; Gina Report, 2006; Coelho-Zanoti, 2007). Por sua vez, a umidade relativa do ar corresponde à razão entre o conteúdo real de umidade de uma amostra de ar e o teor máximo de umidade (saturação) que o mesmo volume de ar pode absorver na mesma temperatura e pressão atmosférica, expressa em porcentagem. Portanto, a umidade relativa indica o grau de saturação do ar e é fortemente influenciada pela temperatura do ar. Assim, a umidade relativa do ar pode variar com a mudança na temperatura do ar, mesmo que não tenha havido nenhum aumento ou diminuição em seu conteúdo de umidade. Os efeitos da precipitação pluviométrica e da temperatura do ar são mais facilmente compreendidos (Ayoade, 2003).

Os estudos de séries temporais epidemiológicas têm sido tradicionalmente utilizados para o estabelecimento de associação entre os efeitos de curto-prazo na saúde e a exposição aos poluentes atmosféricos e as condições climáticas (Botelho et al., 2003; Coelho et al., 2010; Jasinski et al., 2011; Nardocci et al., 2013; Gomez-Acebo et al., 2013). Os mesmos apresentam como vantagens a possibilidade de uso de dados secundários, os quais são de baixo custo e de fácil obtenção. Correlações lineares positivas altamente significativas entre a atividade do VSR e a umidade relativa do ar foram observadas para as cidades de Miami-USA, Cidade do México e Santiago do Chile (Yusuf et al., 2007). Estudos realizados por Falagas et al. (2008) também 
evidenciaram uma correlação positiva entre a incidência de infecções do trato respiratório (ITR) superiores e inferiores e a umidade relativa do ar. Tendência de aumento da morbidade por doenças respiratórias de afecções das vias respiratórias superiores (AVRS) foram observadas com o aumento da temperatura entre os meses de abril e maio na cidade de São Paulo (Gonçalves e Coelho, 2010). Outros estudos relacionando as doenças respiratórias com as variáveis climáticas foram desenvolvidos (Matthew et al., 2008; Meerhoff et al., 2009; Akinyemi e Olusegun, 2011; Bittencourt et al., 2012; Adeboyejo et al., 2012; Edelci e Ribeiro, 2012; Souza et al., 2012).

No Brasil, dados do Ministério da Saúde evidenciam que desde o início do século XX, registra-se aumento de morbimortalidade por doenças do aparelho respiratório. Na última década, a mortalidade por doenças infecciosas e parasitárias caiu de 46\% (em 1930) para 5,3\% (em 2006), as doenças do aparelho circulatório saltaram de $10 \%$ (década de 30 ) para cerca de 30\% (em 2006), enquanto as doenças do aparelho respiratório aumentaram de 13\% (1930) chegando a mais de 30\% em 2003 (Murara et al., 2013).

No estado da Paraíba, o município de Campina Grande (385.213 habitantes, área territorial de $594 \mathrm{~km}^{2}$ e densidade demográfica de 648 hab. $\mathrm{km}^{-2}$ ), caracteriza-se por apresentar temperaturas mais amenas e estação chuvosa regular e bem distribuída no período de março a setembro, enquanto que Monteiro (30.852 habitantes, área territorial de $986,4 \mathrm{~km}^{2}$ e densidade demográfica de 31,2 hab. $\mathrm{km}^{-2}$ ) (IBGE, 2013) se caracteriza por apresentar temperaturas mais elevadas, precipitações pluviais bastante irregulares, com forte variabilidade espacial e temporal, e baixa umidade relativa do ar, exceto nos meses de outono e inverno. Tanto em Campina Grande quanto em Monteiro não são conhecidos trabalhos que relacionem as características climáticas com os casos de incidência de IRA em crianças menores de 2 anos.

Entretanto, os mecanismos pelos quais o aumento da umidade relativa do ar e a diminuição da temperatura do ar representam um aumento das IRA não são totalmente conhecidos. Há variabilidade entre as causas apontadas pelos cientistas e clínicos sobre a contribuição relativa de vários fatores. Entre elas está a transmissibilidade de vários tipos de vírus que afetam a árvore respiratória, os efeitos diretos do frio para a função do sistema respiratório, bem como do sistema imune que conduz a susceptibilidade a infecções e, finalmente, o efeitos indiretos do frio para o comportamento das pessoas, que leva a mudanças epidemiológicas (como superlotação) que permite a transmissibilidade de algumas doenças respiratórias (Falagas et al., 2008; Makinen et al., 2009). Apesar de algumas limitações reconhecidas, como imprecisões de diagnósticos, dentre outras, acredita-se que o presente estudo contribui como informação útil na literatura sobre a associação entre diversas variáveis meteorológicas e IRA. Quanto a aplicabilidade prática, este, juntamente com os resultados de outros estudos pertinentes, podem servir como base para recomendações de modificações comportamentais durante os dias mais frios e úmidos que podem diminuir o efeito das variáveis meteorológicas estudadas sobre a incidência da IRA.

As cidades de Campina Grande e Monteiro são as cidades mais representativas das mesorregiões da Paraíba, Agreste e Borborema, respectivamente. Para estas cidades, existem bancos de dados clínicos e climáticos consistente. Assim, o presente estudo objetivou a avaliação de séries temporais de casos de IRA em crianças menores de dois anos de idade e sua relação com as variáveis climáticas (precipitação pluvial, temperatura e umidade relativa do ar) nos municípios descritos anteriormente.

\section{MATERIAL E MÉTODOS}

\section{1 Área de estudo}

Os dois municípios contemplados neste estudo diferem em localização geográfica e condições climáticas, embora todo o estado da Paraíba, por sua localização dentro da faixa equatorial, é submetido à incidência de alta radiação solar com um grande número de horas de insolação, em virtude da escassa nebulosidade (AESA, 2006). Tal condição determina um clima quente, com pouca variação intra-anual e uma distribuição espacial da temperatura altamente dependente do relevo (Figura 1).

O município de Campina Grande está inserido na unidade geoambiental do Planalto da Borborema, na vertente a barlavento, está incluído na área geográfica de abrangência do semiárido brasileiro. Apesar disso, por estar acima de 500 metros de altitude, possui um clima com temperaturas mais moderadas, considerado Tropical com estação seca (As, de acordo com a classificação climática de Köppen-Geiger). A estação chuvosa se inicia em maio com término em setembro, podendo se estender até outubro.

O município de Monteiro também está inserido na unidade geomorfológica do Planalto da Borborema, na sua vertente sotavento. O clima é do tipo Semiárido, com chuvas de verão, e o período chuvoso se inicia em março com término em abril/maio (Ab'Saber, 2003; CPRM, 2005). Algumas características desses municípios são observadas na Tabela 1 :

\subsection{Dados clínicos e climáticos}

Foram coletados dados no Sistema de Informação de Atenção Básica (SIAB), referentes à IRA em crianças menores de dois anos de idade, para os municípios de Campina Grande e Monteiro, no Estado da Paraíba, fornecidos pelo Departamento 


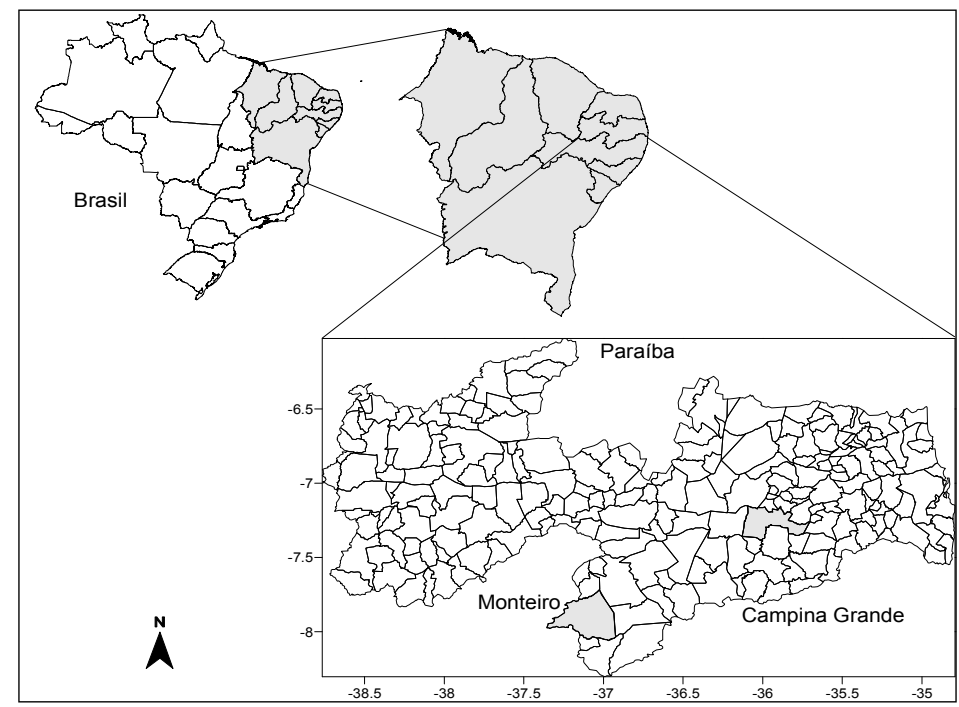

Figura 1 - Localização dos municípios de Campina Grande e Monteiro no estado da Paraíba, na região Nordeste do Brasil.

Tabela 1 - Coordenadas geográficas e valores médios da precipitação pluviométrica anual (Pm), Temperatura (Tm) e umidade relativa (URm) mensais para os municípios selecionados.

\begin{tabular}{lcccccc}
\hline \multicolumn{1}{c}{ Município } & Latitude & Longitude & Altitude $(\mathbf{m})$ & $\begin{array}{c}\mathbf{U R}_{\mathbf{m}} \\
(\mathbf{\%})\end{array}$ & $\begin{array}{c}\mathbf{P}_{\mathbf{m}} \\
(\mathbf{m m})\end{array}$ & $\begin{array}{c}\mathbf{T}_{\mathbf{m}} \\
\left({ }^{\circ} \mathbf{C}\right)\end{array}$ \\
\hline Campina Grande & $7^{\circ} 22^{\prime} \mathrm{S}$ & $35^{\circ} 88^{\prime} \mathrm{O}$ & 547 & 77,7 & 861,4 & 23,5 \\
Monteiro & $7^{\circ} 88^{\prime} \mathrm{S}$ & $37^{\circ} 06^{\prime} \mathrm{O}$ & 603 & 69,4 & 672,6 & 24,5 \\
\hline
\end{tabular}

de Informática do Sistema Único de Saúde (DATASUS), através do site do Ministério da Saúde (http://www2.datasus. gov.br/DATASUS/index.php?area $=02$ ). Foram utilizados dados referentes ao período de 1998 a 2012, correspondem a informações oriundas das Unidades Básicas de Saúde (UBS) dos referidos municípios. Os dados climáticos de precipitação pluvial, temperatura média e umidade relativa do ar, para o período de 1998 a 2012, foram obtidos no site do Instituto Nacional de Meteorologia (INMET) (http://www.inmet.gov.br/portal/), oriundos de estações meteorológicas automáticas e convencionais, registradas junto a Organização Meteorológica Mundial (OMM) sob os números: 82795 (Campina Grande) e 82792 (Monteiro).

\subsection{Tratamento estatístico}

Nesse estudo foi aplicada a regressão linear múltipla, a qual admite que a variável dependente $(y)$ é função de duas ou mais variáveis independentes $\left(x_{j}\right)$. Geralmente, tem-se o interesse em avaliar a relação de uma variável $(y)$ em relação a $k$ variáveis independentes ou covariáveis $\left(x_{j}\right), j=1,2, \ldots, k$. Portanto, do ponto de vista temporal, um possível modelo para avaliar esse tipo de relação pode ser descrito por Hoffmann (2006):

$$
y_{i}=\beta_{0}+\beta_{1} x_{i 1}+\beta_{2} x_{i 2}+\ldots+\beta_{k} x_{i k}+\varepsilon_{i}, i=1, \ldots, n \text {, }
$$

em que, $n$ é o número de intervalos de tempo (indivíduos), $y_{i}$ é a variável dependente para o $i$-ésimo intervalo de tempo, $x_{i}=\left(x_{i 1}, x_{i 2}, \ldots, x_{i k}\right)$ ' é um vetor de observações das variáveis independentes para o $i$-ésimo intervalo de tempo, $\beta=\left(\beta_{0}, \beta_{l}\right.$, $\beta_{2}, \ldots, \beta_{k}$ )' é um vetor de coeficientes de regressão (parâmetros) e $\varepsilon_{i}$ é um componente de erro aleatório. Assumiu-se que esses erros são independentes e seguem distribuição normal com média zero e variância $\left(\sigma^{2}\right)$ constante.

De acordo com Hoffmann (2006), ao estabelecer o modelo de regressão linear múltipla, foram verificados e confirmados, os seguintes pressupostos:

a) A variável dependente $y_{i}$ é uma função linear das variáveis explanatórias $\left(x_{i k}\right)$;

b) Os valores das variáveis explanatórias são fixos;

c) A esperança dos erros $\left(\varepsilon_{i}\right)=0$;

d) Os erros são homocedásticos, isto é, $E\left(\varepsilon^{2}{ }_{i}\right)=\sigma^{2}$;

e) Os erros são não-correlacionados entre si, isto é, $E\left(\varepsilon_{i}\right.$ e $\left.\varepsilon_{j}\right)=0$ para $i \neq j$;

f) Os erros têm distribuição normal.

O coeficiente de determinação da regressão linear múltipla $\left(r^{2}\right)$ e o coeficiente de determinação ajustado $\left(r^{2}{ }_{a}\right)$ foram obtidos por:

$$
r^{2}=\frac{S Q \operatorname{Re} g}{S Q_{T}}=1-\frac{S Q \operatorname{Re} s}{S Q_{T}}
$$




$$
r^{2}{ }_{a}=r^{2}-\frac{n-1}{n-p}\left(1-r^{2}\right)
$$

em que $S Q R e g$ é a soma dos quadrado da regressão; $S Q_{T}$ é a soma dos quadrado total; $n$ é número de observações; $p$ é o número de parâmetros da regressão linear múltipla e, de acordo com os resultados obtidos, foi aplicada a Análise de Variância (ANOVA) e verificado a significância das variáveis independentes através da estatística t-Student (Tabelas 4 e 7). Para verificar se o modelo estava adequado, tendo em vista que, geralmente se admitem como níveis de significância $\alpha=5 \%$ e $\alpha=10 \%$, foram necessários aplicar testes de hipóteses sobre os parâmetros do modelo:

$$
\mathrm{H} 0: \beta 1=\beta 2=\ldots=\beta \mathrm{k}=0
$$

De acordo com Hoffmann (2006), tem-se a distribuição de $F$ com $k$ e $n$ - $p$ graus de liberdade, onde os erros $\varepsilon_{i}$ têm distribuição normal e $\beta_{1}=\beta_{2}=\ldots=\beta_{k}=0$, dada por:

$$
F=\frac{\frac{S Q \operatorname{Re} g}{p}}{\frac{S Q \operatorname{Re} s}{n-p-1}}
$$

Dai,

$$
F_{\text {calc }}=\frac{Q M_{\mathrm{Reg}}}{Q M_{\mathrm{Res}}}
$$

em que $Q M_{\text {Reg }}$ é o quadrado médio da regressão e $Q M_{R e s}$ o quadrado médio dos resíduos. Portanto, se rejeita $H_{0}$ se $F_{c a l c}>F$.

\section{RESULTADOS E DISCUSSÃO}

Foram registradas na cidade de Campina Grande 130.073 casos de infecções respiratórias agudas (IRA) para o período de 1998 a 2012, com média anual de 8.671 casos. O ano de 2004 foi responsável pelo maior registro com 12.865 casos e, o ano de 1998 pelo menor registro com 4.734 casos (Figura 2a). Essa redução observada no número de casos de IRA no ano de 1998 pode estar associada à atuação do fenômeno El Niño nos anos de 1997 e 1998, o qual levou à redução das precipitações sobre o Estado da Paraíba e, consequentemente, elevou a temperatura do ar e reduziu a umidade relativa. A maior média mensal registrada foi no mês de julho com 871 casos, seguida pela média do mês de maio com 844 . O mês de junho apresentou o maior registro de todos os anos (1.344), com

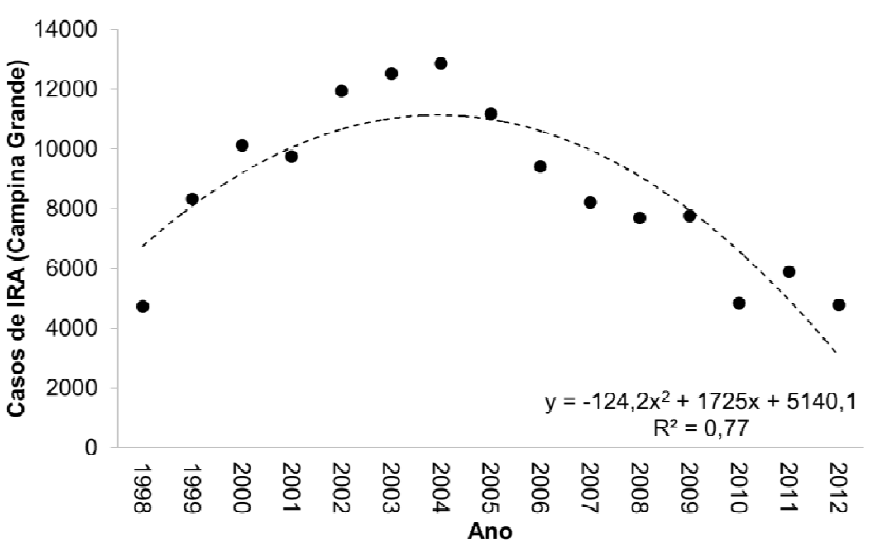

a)

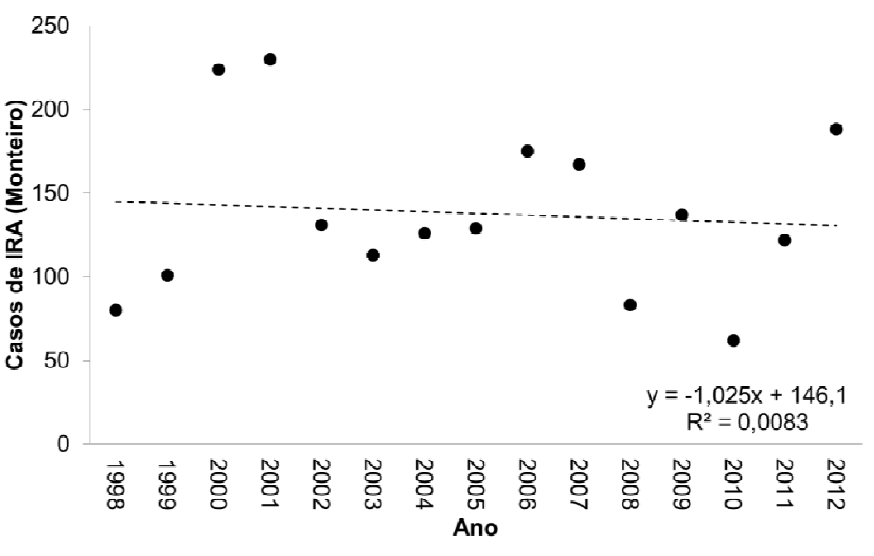

Figura 2 - Distribuição temporal da ocorrência dos casos de infecção respiratória aguda (IRA) em crianças menores de dois anos no período de 1998 a 2012 nos municípios de a) Campina Grande e b) Monteiro, no Estado da Paraíba. 


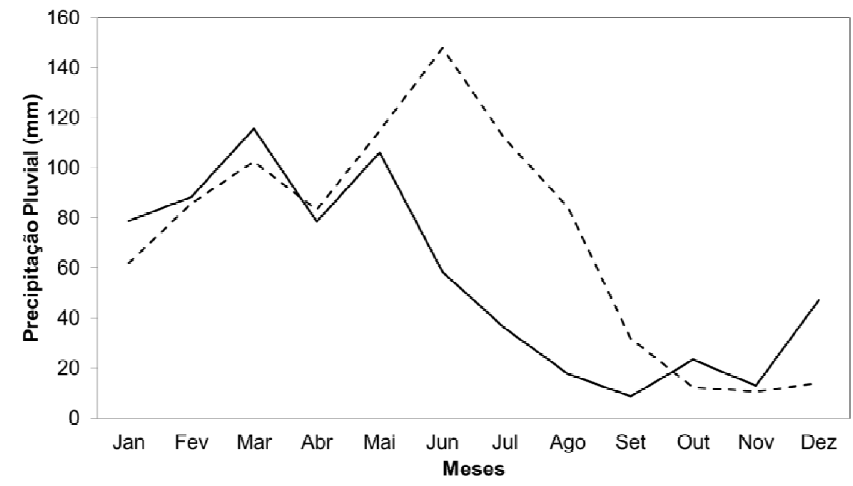

a)
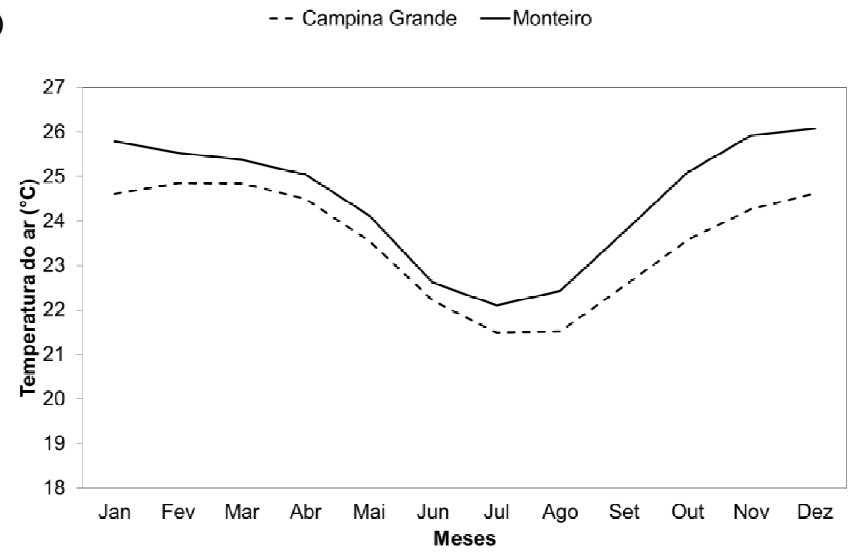

b)
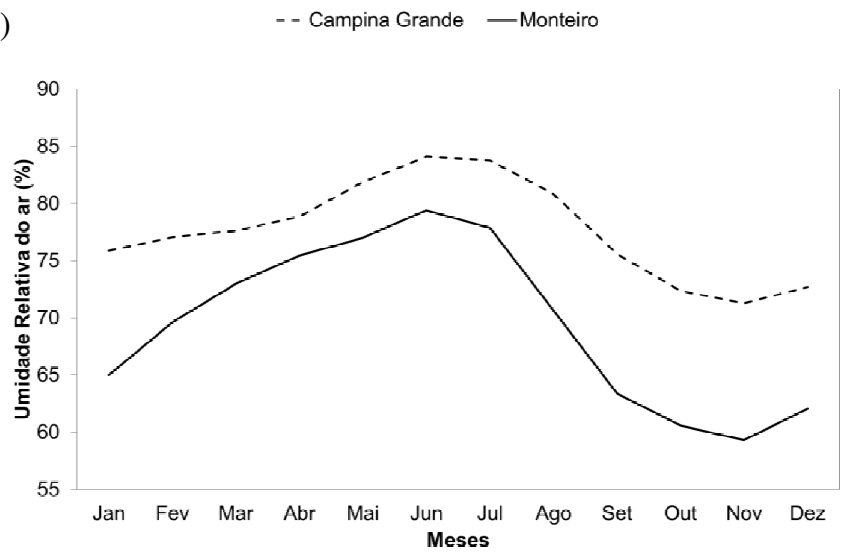

c)

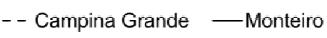

Figura 3 - Comportamento mensal da precipitação pluvial (a), temperatura (b) e umidade relativa do ar (c) para os municípios de Campina Grande e Monteiro.

desvio padrão de 329 casos. O período com maior incidência de casos de IRA coincide com a época do ano da estação chuvosa, a qual se inicia em maio com término em setembro. Durante esse período são registradas as menores temperaturas, com altos valores de umidade relativa e maior concentração de precipitação (ver Figura 3). A menor média mensal verificada ocorreu nos meses de dezembro e janeiro (Tabela 2). Isto se explica pelo fato destes meses apresentarem temperaturas mais elevadas e umidade relativa do ar mais baixa, visto que se trata da estação de verão.
No município de Monteiro, durante o período de estudo, foram registrados 2.068 casos de IRA, com média anual de internações de 141,7 casos. O ano de 2000 foi responsável pelo maior registro com 224 casos e, o ano de 2010 pelo menor registro com 62 casos (Figura 2b). A maior média mensal registrada foi no mês de julho com 17,4 casos, seguida pela média do mês de abril com 14,4. O mês de julho apresentou o maior registro de casos de todos os anos (14) com desvio padrão de 9,1 casos. A menor média mensal verificada ocorreu nos meses de dezembro e janeiro (Tabela 3). 
Tabela 2 - Número e estatísticas de crianças menores de dois anos com infecção respiratória aguda (IRA) no período de 1998 a 2012 para o município de Campina Grande-PB.

\begin{tabular}{|c|c|c|c|c|c|c|c|c|c|c|c|c|c|}
\hline & \multicolumn{13}{|c|}{ Meses } \\
\hline & Jan & Fev & Mar & Abr & Mai & Jun & Jul & Ago & Set & Out & Nov & Dez & Total \\
\hline Média & 598 & 617 & 683 & 762,1 & 844 & 824 & 871 & 780 & 711 & 705 & 684 & 588 & 8.671 \\
\hline Min. & 156 & 308 & 251 & 313 & 239 & 215 & 396 & 396 & 224 & 381 & 133 & 266 & 3.278 \\
\hline Max. & 1.031 & 996 & 1.177 & 1.231 & 1.256 & 1.377 & 1.344 & 1.161 & 1.141 & 1.186 & 1.097 & 869 & 13.866 \\
\hline D.P. & 221 & 183 & 227 & 278 & 355 & 329 & 292 & 234,5 & 254 & 237 & 272 & 208 & 3.095 \\
\hline Total & 8.979 & 9.263 & 10.251 & 11.431 & 12.660 & 12.362 & 13.069 & 11.702 & 10.671 & 10.586 & 10.269 & 8.830 & 130.073 \\
\hline
\end{tabular}

Tabela 3 - Número e estatísticas de crianças menores de dois anos com infecção respiratória aguda (IRA) no período de 1998 a 2012 para o município de Monteiro-PB.

\begin{tabular}{lccccccccccccc}
\hline & \multicolumn{10}{c}{ Meses } & \multicolumn{10}{c}{} \\
\cline { 2 - 14 } & Jan & Fev & Mar & Abr & Mai & Jun & Jul & Ago & Set & Out & Nov & Dez & Total \\
\hline Média & 8,3 & 11,2 & 10,5 & 14,4 & 12,1 & 14,2 & 17,4 & 12,7 & 10,7 & 9,7 & 11,6 & 8,9 & 141,7 \\
Min. & 1 & 3 & 3 & 5 & 2 & 5 & 2 & 3 & 2 & 4 & 3 & 2 & 35,0 \\
Máx. & 20 & 27 & 17 & 25 & 22 & 39 & 33 & 34 & 29 & 19 & 36 & 19 & 320,0 \\
D.P. & 5,7 & 7,3 & 4,4 & 7,0 & 6,4 & 8,8 & 9,1 & 8,1 & 6,8 & 4,7 & 8,3 & 4,8 & 81,4 \\
Total & 124 & 146 & 158 & 201 & 182 & 213 & 261 & 190 & 160 & 136 & 163 & 134 & 2.068 \\
\hline
\end{tabular}

Tabela 4 - Análise de variância (ANOVA) para o modelo de regressão linear múltipla ajustado aos dados do município de Campina Grande - PB.

\begin{tabular}{lcccc}
\hline & Coeficientes & Erro padrão & t-Student & p-valor \\
\hline Temperatura & $-19,772$ & 6,302 & $-3,137$ & 0,011 \\
Umidade relativa & 15,290 & 1,908 & 8,012 & $<0,001$ \\
\hline
\end{tabular}

Tanto para o município de Campina Grande, quanto para Monteiro, os maiores valores de ocorrência de IRA foram observados nos meses de junho e julho, respectivamente. Esse resultado corrobora com aqueles obtidos por Nardocci et al. (2013), onde as doenças respiratórias em crianças menores de 5 anos apresentaram padrão de distribuição com sazonalidade característica, tendo maior frequência nos meses de inverno.

Com relação às variáveis climáticas, observa-se que a estação chuvosa ocorre em períodos distintos nestes municípios, compreendendo os meses de maio, junho e julho em Campina Grande e fevereiro, março e abril em Monteiro (Figura 3a). Quanto à temperatura, os valores mais elevados, durante todos os meses do ano, são observados em Monteiro (Figura 3b). Já com relação aos valores de umidade relativa do ar, Campina Grande apresenta valores mais elevados (Figura 3c). Observa-se que as variáveis temperatura e umidade relativa do ar apresentam comportamentos que acompanham as estações do ano, com temperaturas mais elevadas na primavera/verão e umidade relativa do ar mais baixa no outono/inverno (Figuras $3 \mathrm{~b}$ e $3 \mathrm{c}$ ).

A análise das correlações entre os casos de IRA e as variáveis climáticas (precipitação pluvial, temperatura e umidade do ar) foi importante para avaliar as correlações inversas e diretas, sendo possível associar os efeitos das condicionantes climáticas na saúde humana. Para o município de Campina Grande (Tabela 4), pode-se observar que apenas as variáveis independentes (temperatura e umidade relativa do ar) foram estatisticamente significativas, ao nível de significância de $5 \%(\mathrm{p}<0,05)$, para explicar a variabilidade da incidência de IRA. É importante ressaltar que o intercepto não foi significativo $(p<0,05)$. Conforme o coeficiente de determinação $\left(r^{2}\right), 99 \%$ da variação total foi explicada pelo modelo de regressão linear múltipla (Tabela 5), onde foram verificados os pressupostos do modelo utilizando-se o teste de Shapiro Wilk e o QQ - plot (Figura 4a). As análises foram realizadas por meio do Software R (R Core Team, 2014).

Convém ressaltar que na análise de variância (ANOVA) para o modelo de regressão linear múltipla, ajustado aos dados do município de Campina Grande - PB, a variável precipitação pluvial foi considerada pouco relevante, por não explicar as ocorrências de IRA, uma vez que foi observada significância apenas entre as variáveis climáticas temperatura e umidade relativa (variáveis independentes) com as referidas infecções (variável dependente) (Tabela 4). A matriz de correlação evidencia a correlação linear múltipla entre as variáveis analisadas (Tabela 6). Observa-se que a umidade relativa ( $\rho=$ $0,79)$ e temperatura do ar $(\rho=-0,74)$ apresentam-se fortemente correlacionadas com a ocorrência das doenças. Esses resultados indicam que, à medida que a umidade relativa do ar aumenta, ocorre um aumento no número de casos de IRA. Comportamento inverso pode ser observado para temperatura do ar, ou seja, à 

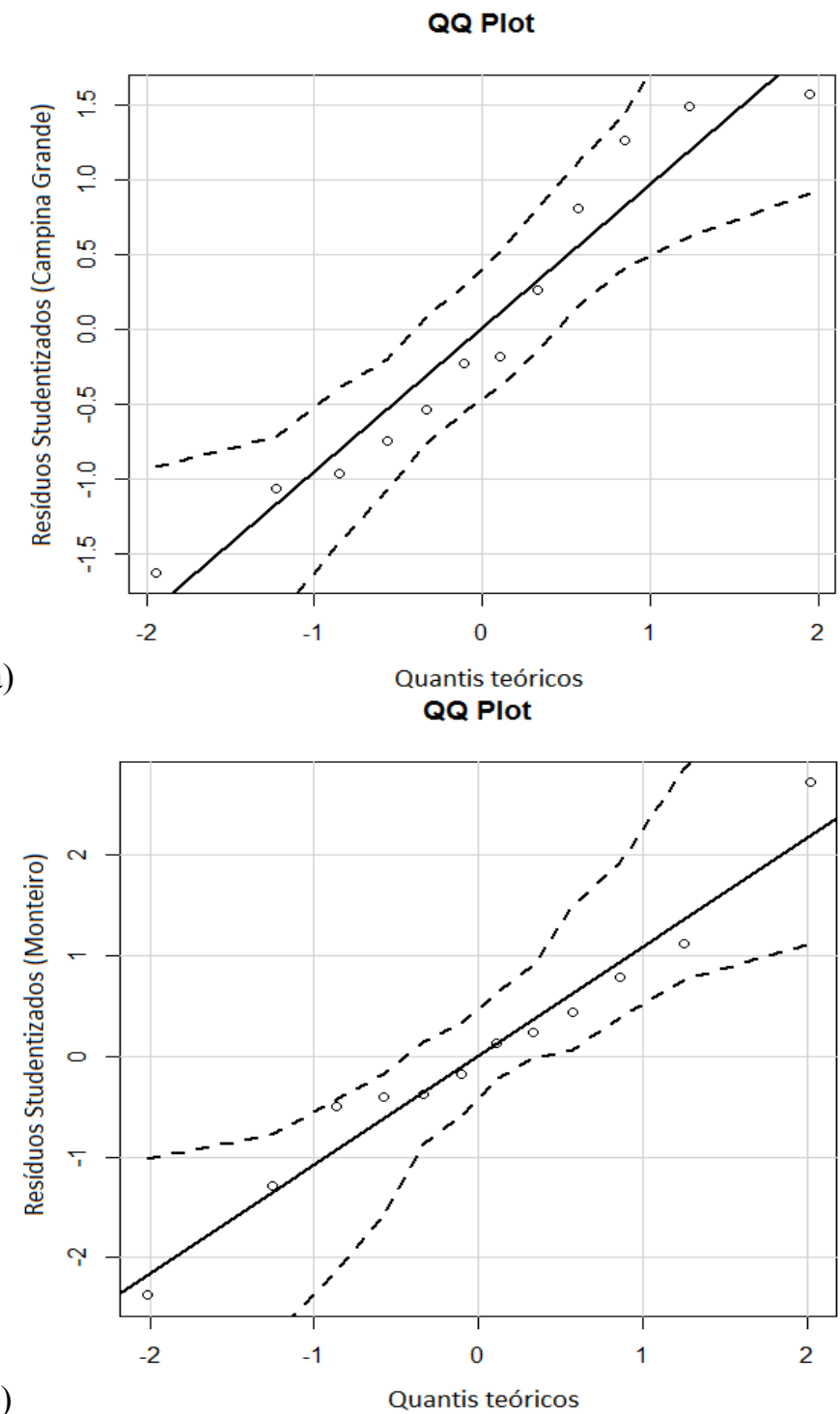

Figura 4 - Envelope de simulação com os resíduos studentizados versus quantis teóricos sob normalidade representados pelo QQ-plot para o município de a) Campina Grande e b) Monteiro, no Estado da Paraíba.

Tabela 5 - Parâmetros da correlação dos fatores meteorológicos em conjunto com a ocorrência de infecção respiratória aguda (IRA) em crianças menores de dois anos para o município de Campina Grande e Monteiro.

\begin{tabular}{lcc}
\multicolumn{1}{c}{ Parâmetros } & Campina Grande & Monteiro \\
\hline $\mathrm{r}^{2}$ & 0,9962 & 0,9059 \\
$\mathrm{r}^{2}$ & 0,9955 & 0,8706 \\
Shapiro-Wilk (W) & 0,9309 & 0,9834 \\
p-valor & 0,3895 & 0,9938 \\
\hline
\end{tabular}

Tabela 6 - Matriz de correlação de Pearson para o município de Campina Grande.

\begin{tabular}{lcccc}
\hline & Doença & Precipitação & Temperatura & Umidade relativa \\
\hline Doença & 1,00 & & & \\
Precipitação & 0,63 & 1,00 & & \\
Temperatura & $-0,74$ & $-0,34$ & 1,00 & 1,00 \\
Umidade relativa & 0,79 & 0,93 & $-0,60$ & \\
\hline
\end{tabular}


medida que se reduz a temperatura do ar, ocorre aumento nos casos de IRA.

A equação obtida com a regressão linear múltipla foi: $\hat{y}=-19,77 x_{1}+15,29 x_{2}$. Assim, para uma temperatura fixa, cada incremento na unidade de temperatura corresponde a uma redução de, aproximadamente, 20 casos de IRA. E para valores de umidade relativa do ar fixos, cada incremento na unidade de umidade relativa do ar corresponde a um aumento de, aproximadamente, 15 casos de IRA.

De acordo com a ANOVA para o modelo de regressão linear múltipla ajustado aos dados no município de Monteiro, pode-se observar que todas as variáveis independentes foram estatisticamente significativas, ao nível de significância 5\% $(\mathrm{p}<0,05)$, para explicar a variabilidade da incidência de IRA (Tabela 7). Conforme o coeficiente de determinação $\left(\mathrm{r}^{2}\right), 91 \%$ da variação total foi explicada pelo modelo de regressão linear múltipla (Tabela 5), onde foram verificados os pressupostos do modelo utilizando-se o teste de Shapiro Wilk e o QQ-plot (Figura 4b). A equação obtida foi: $\hat{y}=-66,60-0,09 x_{1}+1,47 x_{2}+0,68 x_{3}$, ou seja, para valores de temperatura e umidade fixos, a cada acréscimo de unidade de precipitação, há um decréscimo de aproximadamente 0,1 no número de casos de IRA.

Como se pode observar na Tabela 8 , a umidade relativa $(\rho=0,73)$ e temperatura do ar $(\rho=-0,73)$ apresentam-se fortemente correlacionadas com a ocorrência de IRA. Indicando assim, que aumento na umidade relativa do ar eleva o número de casos de IRA. Sendo observado comportamento inverso para a temperatura do ar. Correlações lineares positivas altamente significativas entre a incidência de doenças respiratórias e as variáveis climáticas (temperatura e umidade relativa do ar) foram observadas por (Yusuf et al., 2007) para o VSR, (Falagas et al., 2008) para a incidência de infecções do trato respiratório (ITR) superiores e inferiores e Gonçalves e Coelho (2010) para as afecções das vias respiratórias superiores (AVRS). Outros resultados similares foram obtidos por Matthew et al. (2008), Meerhoff et al. (2009), Akinyemi e Olusegun (2011), Bittencourt et al. (2012), Adeboyejo et al. (2012), Edelci e Ribeiro (2012) e Souza et al. (2012).

\section{CONCLUSÕES}

Com base na análise dos dados climáticos e clínicos para os municípios de Campina Grande e Monteiro, no Estado da Paraíba, é possível concluir que:

1) Para Campina Grande evidencia-se um comportamento quadrático da variável dependente (IRA) em crianças menores de dois anos. Observa-se, ainda, que a ocorrência máxima desses casos está compreendida entre os anos de 2003 e 2005, sendo que, a partir desse período, houve um decréscimo nos casos de IRA. Provavelmente, esse comportamento deve-se às melhorias nas condições de vida da população nesse período, bem como às campanhas preventivas e informativas, diagnósticos mais eficazes e tratamentos mais adequados. No entanto, para Monteiro, o número anual de casos de ocorrência de IRA em crianças menores de dois anos é bastante variável e não apresenta períodos distintos e regulares de ocorrências como os registrados em Campina Grande;

2) Os municípios de Monteiro e Campina Grande têm as maiores ocorrências de IRA nos meses de junho e julho, respectivamente, apresentando padrão de distribuição com característica sazonal, tendo maior frequência nos meses de inverno.

3) As variáveis climáticas evidenciaram estações chuvosas diferenciadas para os dois municípios, estendendo-se de maio a julho em Campina Grande e de fevereiro a abril em Monteiro, sendo a precipitação pluvial à variável menos uniforme e praticamente não correlacionada com os casos de ocorrência de IRA em crianças menores de dois anos em Campina Grande;

4) Analisando a influência conjunta das variáveis climáticas (precipitação pluvial, temperatura e umidade relativa do ar), observa-se que, para Campina Grande, apenas

Tabela 7 - Análise de variância (ANOVA) para o modelo de regressão linear múltipla ajustado aos dados do município de Monteiro - PB.

\begin{tabular}{lcccc}
\hline & Coeficientes & Erro padrão & t-Student & p-valor \\
\hline Intercepto & $-66,599$ & 21,046 & $-3,164$ & 0,013 \\
Precipitação & $-0,090$ & 0,020 & $-4,527$ & 0,002 \\
Temperatura & 1,467 & 0,560 & 2,620 & 0,031 \\
Umidade relativa & 0,684 & 0,125 & 5,454 & 0,001 \\
\hline
\end{tabular}

Tabela 8 - Matriz de correlação de Pearson para o município de Monteiro

\begin{tabular}{lcccc}
\hline \multicolumn{1}{c}{ Variável } & Doença & Precipitação pluvial & Temperatura & Umidade relativa \\
\hline Doença & 1,00 & & & \\
Precipitação & $-0,08$ & 1,00 & & \\
Temperatura & $-0,73$ & 0,29 & 1,00 & 1,00 \\
Umidade relativa & 0,73 & 0,51 & $-0,62$ & \\
\hline
\end{tabular}


a temperatura e a umidade relativa do ar foram significativas para representar a incidência de IRA em crianças menores de 2 anos, com 99\% da variabilidade explicada pelo modelo ajustado aos dados. Já para Monteiro, a análise mostra que as três variáveis climáticas foram significativas para representar a incidência dessas infecções, com $90 \%$ da variabilidade explicada pelo modelo.

\section{REFERÊNCIAS}

AB' SABER, A. N. Os domínios de Natureza no Brasil: potencialidades paisagísticas. 2. ed. São Paulo: Ateliê Editorial, 2003. 158 p.

ADEBOYEJO, A. T.; LIRVHUWANI, M.; SHONISANI, D. K. Impact of Climate Change on Children's Health in Limpopo Province, South Africa. International. Journal of Environmental Research and Public Health, v. 9, p. 831-854, 2012.

AESA - AGÊNCIA EXECUTIVA DE GESTÃO DAS ÁGUAS DO ESTADO DA PARAIIBA. Plano Estadual de Recursos Hídricos - Resumo Executivo. SEMARH/AESA. João Pessoa - PB. Disponível em CD-ROM, 2006, 255 p.

AKINYEMI, G. O.; OLUSEGUN, O. A. Study of Weather Related Respiratory Diseases in Eco-climatic Zones. African Physical Review, v. 5, n. 3, p. 41-56, 2011.

ANDREOLI, T. E.; CARPENTER, C. C. J.; GRIGGS, R. C.; LOSCALZO, J. Medicina Interna Básica - Doenças Respiratórias, $1993,400 \mathrm{p}$.

AYOADE, J. O. Introdução a Climatologia para os Trópicos. 10. ed. Rio de Janeiro: Bertrand Brasil 2003, 332 p.

BENGUIGUI, Y. As infecções respiratórias agudas na infância como problema de saúde pública. Boletim de Pneumologia Sanitária, v. 10, n. 1, p. 13-22, 2002.

BITTENCOURT, P. R. G.; BERGER, R.; LUCAMBIO, F. P.; LARA, P. T. P. Relações entre as doenças respiratórias e a poluição atmosférica e variáveis climáticas na cidade de Curitiba, Paraná, Brasil. Revista de Saúde Pública, v. 42, n. 4, p. $817-828,2012$.

BOTELHO, C.; CORREIA, A. L.; SILVA, A. M. C.; MACEDO, A. G.; SILVA, C. O. S. Fatores ambientais e hospitalizações em crianças menores de cinco anos com infecção respiratória aguda. Cadernos de Saúde Pública, v. 19, n. 6, p. 17711780, 2003.

CARDOSO, M. R. Micro-Clima dos domicílios de doenças sibilantes em crianças da cidade de São Paulo. Tese (Doutorado) Faculdade Saúde Pública - Universidade de São Paulo, São Paulo 2007, 190 p.

COELHO, M. S. Z. S.; GONÇALVES, F. L. T.; LATORRE, M. do R. D. de O. Statistical analysis aiming at predicting respiratory tract disease hospital admissions from environmental variables in the city of São Paulo. Journal of Environmental and Public Health, v. 2010, p. 1-11, 2010. COELHO-ZANOTI, M. S. S. Uma análise estatística com vistas a previsibilidade de internações por doenças respiratórias em função das condições meteorológicas na cidade de São Paulo. Tese (Doutorado em Ciências Atmosféricas), Universidade de São Paulo - USP, São Paulo, 2007, 178 p.

CPRM. SERVIÇO GEOLÓGICO DO BRASIL - Projeto Cadastro de Fontes de abastecimento por água subterrânea - Diagnóstico de Fontes do município de Monteiro e Campina Grande/ PB. CPRM/ PRODEEM, 2005, 19 p.

EDELCI, N. S. e RIBEIRO, H. Impact of urban atmospheric environment on hospital admissions in the elderly. Revista de Saúde Pública, v. 46, n. 4, p. 694-701, 2012.

FALAGAS, M. E.; THEOCHARIS, G.; SPANOS, A.; VLARA, L. A.; ISSARISE, E. A.; PANOS G.; PEPPAS, G. Effect of meteorological variables on the incidence of respiratory tract infections. Respiratory Medicine, 2008, v. 102, p. 733-737.

GINA REPORT. Global Strategy for Asthma Management and Prevention - Asthma Management and prevention Chapter 4 - The GINA reports, 2006.

GOMEZ-ACEBO, I.; LLORCA, J.; DIERSSEN, T. Cold-related mortality due to cardiovascular diseases, respiratory diseases and cancer: a case-crossover study. Public Health, v. 127, p. $252-258,2013$.

GOLÇALVES, F. L. T.; COELHO, M de S. Z. S. Variação da morbidade de doenças respiratórias em função da variação da temperatura entre os meses de abril e maio em São Paulo. Ciência e Natura, v. 32, n. 1, p. 103-118, 2010.

HOFFMANN, R. Análise de Regressão: Uma Introdução á Econometria. São Paulo: HUCITEC, 2006, 378 p.

IBGE. INSTITUTO BRASILEIRO DE GEOGRAFIA E ESTATÍSTICA. Censo 2010. http://censo2010.ibge.gov.br/ (acessado em 28/Set/2013).

JASINSKI, R.; PEREIRA L. A.; BRAGA, A. L. F.; Poluição atmosférica e internações hospitalares por doenças respiratórias e crianças e adolescentes em Cubatão, São Paulo, Brasil, entre 1997-2004. Cadernos de Saúde Pública, v. 27, p. 2242-52, 2011.

MATTHEW, E. F.; GEORGE, T.; ALEX, S.; LAMBRINI, A. V.; EVANGELOS, A. I.; GEORGE, P. Effect of meteorological variables on the incidence of respiratory tract infections. Respiratory Medicine, v. 102, p. 733-737, 2008.

MAKINEN, T. M.; JUVONEN, R.; JOKELAINEN, J.; HARJU, T. H.; PEITSO, A.; BLOIGU, A.; SILVENNOINENKASSINEN, S.; LEINONEN, M.; HASSI, J. Cold temperature and low humidity are associated with increased 
occurrence of respiratory tract infections. Respiratory Medicine, v. 103, p. 456 - 462, 2009,

MEERHOFF, T. J.; PAGET, W. J.; KIMPEN, J. L.; SCHELLEVIS, F. Variation of respiratory syncytial virus and the relation with meteorological factors in different winter seasons. Pediatric Infectious Disease Journal, v. 28, n. 10, p. 860- 866, 2009.

MURARA, P. G.; MENDONÇA, M.; BONETTI, C. O clima e as doenças circulatórias e respiratórias em Florianópolis/ SC. Hygeia, v. 9, n. 16, p. $86-102,2013$.

NARDOCCI, A. C.; FREITAS, C. U.; PONCE DE LEON, A. C. M.; JUNGER, W. L. e GOUVEIA, N. C. Poluição do ar e doenças respiratórias e cardiovasculares: estudo de séries temporais em Cubatão, São Paulo, Brasil. Cadernos de Saúde Pública, v. 29, n. 9, p. 1867-1876, 2013.

OMS. ORGANIZAÇÃO MUNDIAL DA SAÚDE. World Health Report 2002: Reducing Risks, Promoting Healthy Life. Geneva: World Health Organization 2002, 247 p.

OMS. ORGANIZAÇÃO MUNDIAL DA SAÚDE. Changing history. Geneva: 2004, 170 p.
R CORE TEAM. R: A language and environment for statistical computing. R Foundation for Statistical Computing, Vienna, Austria. URL http://www.R-project.org/. 2014.

RUDAN, I.; BOSCHI-PINTO, C.; BILOGLAV, Z.; MULHOLLAND, K.; CAMPBELL, H. Epidemiology and etiology of childhood pneumonia. Bulletin of the World Health Organization, v. 86, p. 408-16, 2008.

SOUZA, A, FERNANDES, W. A.; PAVÃO, H. G.; LASTORIA, G.; ALBREZ, E. A. Potential impacts of climate variability on respiratory morbidity in children, infants, and adults. Journal Brasileiro de Pneumologia, v. 38, n. 6, p. 708715, 2012.

YUSUF, S.; PIEDIMONTE, G. A.; AUAIS, A.; DEMMLER, G.; KRISHNAN, S.; VAN CAESEELE, P.; SINGLETON, R.; BROOR, S.; PARVEEN, S.; AVENDANO, L.; PARRA, J.; CHAVEZ-BUENO, S.; MURGUÍA DE SIERRA, T.; SIMOES, E. A. F.; SHAHA, S.; WELLIVER, R. The relationship of meteorological conditions to the epidemic activity of respiratory syncytial virus. Epidemiology and Infection, v. 135, p. 1077-1090, 2007. 